\begin{tabular}{|c|l|}
\hline Title & $\begin{array}{l}\text { Derivation of the electron drift velocity vector in gas under crossed ac electric and dc magnetic fields assuming } \\
\text { constant-collision-frequency models }\end{array}$ \\
\hline Author(s) & Sugawara, Hirotake \\
\hline Citation & $\begin{array}{l}\text { Japanese Journal of A pplied Physics, 58(10), 108002-1-108002-4 } \\
\text { https://doi.org/10.7567/1347-4065/ab3e5d }\end{array}$ \\
\hline Issue Date & 2019-09-12 \\
\hline Doc URL & http://hdl.handle.net/2115/79254 \\
\hline Rights & @2019 The Japan Society of A pplied Physics \\
\hline Type & article (author version) \\
\hline File Information & Sugawara 2019-JJA P-58(10)108002-HUSCA P.pdf \\
\hline
\end{tabular}

Instructions for use 


\title{
Derivation of the electron drift velocity vector in gas under crossed ac electric and dc magnetic fields assuming constant-collision-frequency models *
}

\author{
Hirotake SUGAWARA ${ }^{\dagger}$ \\ Graduate School of Information Science and Technology, Hokkaido University, Sapporo 060-0814, \\ Japan
}

The periodic variation of the average electron velocity vector under crossed ac electric and dc magnetic fields is formulated analytically on the basis of kinetic equations for single-electron motion assuming constant-collision-frequency models with and without ionization. The velocity vector draws elliptic loci with the alternation of the electric field, and this is verified by Monte Carlo simulations. The amplitude and the mean deflection angle of the velocity vector, which represent basic features of electron transport in magnetized plasmas, are explicitly described with the collision frequency, the ac angular frequency, and the intensities of the fields.

KEYWORDS: electron drift velocity, vector locus, constant-collision-frequency model, crossed electric and magnetic fields

Electron transport in gas under crossed electric and magnetic fields $(\boldsymbol{E} \times \boldsymbol{B}$ fields $)$ is a key property to govern the structure and response of magnetized inductively coupled plasmas (ICPs) used for material processing ${ }^{1-5}$ and ion sources. ${ }^{6,7}$ The microscopic electron behavior in $\boldsymbol{E} \times \boldsymbol{B}$ fields has been simulated from various viewpoints - e.g., the rf response of electron swarms,${ }^{8-11}$ plasma structure formation, ${ }^{12-18}$ electron conduction directionality, ${ }^{19,20}$ power deposition, ${ }^{21-26}$ and magnetic shutter and filter effects. ${ }^{27-29}$ Electron transport coefficients are also of particular interest for deeper understanding and desirable control of magnetized plasmas. However, techniques to derive or estimate their direction dependent values under the $\boldsymbol{E} \times \boldsymbol{B}$ fields that fluid simulations demand are still under development. ${ }^{30-32}$ A recent effort $^{33}$ showed that the vector $\boldsymbol{V}$ of the average electron velocity in Ar under ac electric and dc magnetic fields draws elliptic loci. This visual depiction is informative for observing the characteristics of the electron swarm response depending on the field conditions. For example, the amplitude of $\boldsymbol{V}$ and the temporal variation of the Hall deflection angle can be understood at a glance. In this report, $\boldsymbol{V}$ is derived analytically assuming constant-collision-frequency (CCF) model gases with and without ionization. Although this is an ideal model, a real gas $\mathrm{F}_{2}$ has a CCF-like feature, ${ }^{34}$ and it is of significant merit that the explicit description of $\boldsymbol{V}$ using the collision frequency and the field parameters enables us to estimate the basic

\footnotetext{
*Published source: Japanese Journal of Applied Physics, Vol. 58, No. 10, 108002-1-108002-4 (2019),

DOI: $10.7567 / 1347-4065 / \mathrm{ab3e} 5 \mathrm{~d}$

${ }^{\dagger}$ E-mail: sugawara@ist.hokudai.ac.jp
} 
tendency of the electron drift under $\boldsymbol{E} \times \boldsymbol{B}$ fields with theoretical grounds.

The ac electric field $\boldsymbol{E}(t)$ and the dc magnetic field $\boldsymbol{B}$ are defined to be uniform in boundary-free space as

$$
\begin{aligned}
\boldsymbol{E}(t) & =\left(0,-E \sin \left(\omega_{E} t+\phi\right), 0\right) \quad(E>0), \\
\boldsymbol{B} & =(0,0, B) \quad(B>0),
\end{aligned}
$$

where $\omega_{E}=2 \pi f$, and $f$ and $\phi$ are the frequency and initial phase of $\boldsymbol{E}(t)$, respectively. This configuration $\boldsymbol{E} \perp \boldsymbol{B}$ often appears in the above-mentioned magnetized ICPs and their simulations. The equation of motion for an electron with velocity $\boldsymbol{v}=\left(v_{x}, v_{y}, v_{z}\right)$ is

$$
d \boldsymbol{v} / d t=-(e / m)[\boldsymbol{E}(t)+\boldsymbol{v} \times \boldsymbol{B}],
$$

where $e$ and $m$ are the electronic charge and mass, respectively. The components $v_{x}, v_{y}$, and $v_{z}$ satisfy

$$
\begin{aligned}
d v_{x} / d t & =-(e / m) v_{y} B=-\omega_{B} v_{y}, \\
d v_{y} / d t & =-(e / m)\left[-E \sin \left(\omega_{E} t+\phi\right)-v_{x} B\right] \\
& =\omega_{B} v_{x}+\omega_{E} v_{E} \sin \left(\omega_{E} t+\phi\right), \\
d v_{z} / d t & =0,
\end{aligned}
$$

where $\omega_{B}=e B / m>0$ and $v_{E}=e E /\left(m \omega_{E}\right)>0$.

Single-electron motion during a collisionless flight has the following two sets of analytical solutions. With a given initial velocity $\boldsymbol{v}_{0}=\left(v_{x 0}, v_{y 0}, v_{z 0}\right)$ at $t=0$, the non-resonant solution for $\omega_{E} \neq \omega_{B}$ is given as

$$
\begin{aligned}
v_{x}(t)= & {\left[v_{x 0}+\left(\omega_{B} \omega_{E} / \Omega\right) v_{E} \sin \phi\right] \cos \omega_{B} t } \\
& -\left[v_{y 0}-\left(\omega_{E}^{2} / \Omega\right) v_{E} \cos \phi\right] \sin \omega_{B} t \\
& -\left(\omega_{B} \omega_{E} / \Omega\right) v_{E} \sin \left(\omega_{E} t+\phi\right), \\
v_{y}(t)= & {\left[v_{x 0}+\left(\omega_{B} \omega_{E} / \Omega\right) v_{E} \sin \phi\right] \sin \omega_{B} t } \\
& +\left[v_{y 0}-\left(\omega_{E}^{2} / \Omega\right) v_{E} \cos \phi\right] \cos \omega_{B} t \\
& +\left(\omega_{E}^{2} / \Omega\right) v_{E} \cos \left(\omega_{E} t+\phi\right), \\
v_{z}(t)= & v_{z 0}, \\
\Omega= & \left(\omega_{B}+\omega_{E}\right)\left(\omega_{B}-\omega_{E}\right) .
\end{aligned}
$$

On the other hand, the resonant solution at $\omega_{E}=\omega_{B} \equiv \omega$, at which the ECR occurs, is

$$
\begin{aligned}
v_{x}(t)=\quad\left(v_{x 0} \cos \phi+v_{y 0} \sin \phi\right. \\
\left.\quad+\frac{1}{2} v_{E} \sin \phi \cos \phi+\frac{1}{2} v_{E} \omega t\right) \cos (\omega t+\phi)
\end{aligned}
$$




$$
\begin{aligned}
+ & \left(v_{x 0} \sin \phi-v_{y 0} \cos \phi\right. \\
& \left.-\frac{1}{2} v_{E} \cos ^{2} \phi\right) \sin (\omega t+\phi), \\
v_{y}(t)= & \left(v_{x 0} \cos \phi+v_{y 0} \sin \phi\right. \\
& \left.+\frac{1}{2} v_{E} \sin \phi \cos \phi+\frac{1}{2} v_{E} \omega t\right) \sin (\omega t+\phi) \\
- & \left(v_{x 0} \sin \phi-v_{y 0} \cos \phi\right. \\
& \left.+\frac{1}{2} v_{E} \sin ^{2} \phi\right) \cos (\omega t+\phi), \\
v_{z}(t)= & v_{z 0} .
\end{aligned}
$$

$\boldsymbol{V}=\left(V_{x}, V_{y}, V_{z}\right)$ of the electron swarm is defined as

$$
\boldsymbol{V} \equiv\left(\bar{v}_{x}, \bar{v}_{y}, \bar{v}_{z}\right) \equiv\left(V_{\boldsymbol{E} \times \boldsymbol{B}}, V_{\boldsymbol{E}}, V_{\boldsymbol{B}}\right),
$$

where $\bar{X}$ represents the average of a quantity $X$ among the electrons under consideration. Here, $V_{z}=0$ for the reflective symmetry of the electron velocity distribution function (EVDF) with respect to the $v_{x} v_{y}$-plane. $V_{x}$ and $V_{y}$ are derived in a similar way to that under dc $\boldsymbol{E} \times \boldsymbol{B}$ fields. ${ }^{34}$ Hereafter, it is assumed that the total collision frequency $\nu$ is constant irrespective of $v=|\boldsymbol{v}|$ and the electron scattering is isotropic.

Let us define an electron squad as a group of electrons that undergo scattering at the same time $t^{\prime}$ and start their flight together. First, $\overline{\boldsymbol{v}}_{0}=\overline{\boldsymbol{v}}\left(t^{\prime}\right)=0$ because the scattering is isotropic; the initial EVDF of the squad is centered at $\boldsymbol{v}=0$. Next, the Coulomb and Lorentz forces induce a concentric rotation of the EVDF, but its shape is kept isotropic around its center, which moves in the same way as a single electron. Furthermore, even in the presence of collisions, the EVDF of the squad decays exponentially keeping its shape isotropic around its center because the dropout of electrons by scattering occurs uniformly from the squad in the CCF condition. Therefore, the $\overline{\boldsymbol{v}}(t)$ of the squad follows the same temporal variation as the $\boldsymbol{v}(t)$ of the single electron motion starting from $\boldsymbol{v}_{0}=0$ at $t=t^{\prime} \cdot \boldsymbol{V}(t)$ is obtained by integrating $\overline{\boldsymbol{v}}(t)$ [substitutable with $\boldsymbol{v}(t)$ ] of all squads with the weight of the electron population.

The practical $\bar{v}_{x}(t)=v_{x}(t)$ and $\bar{v}_{y}(t)=v_{y}(t)$ of a squad are obtained by substituting the flight time $t-t^{\prime}$ for $t$ and the initial phase $\omega_{E} t^{\prime}+\phi$ for $\phi$ in Eqs. (7)-(13) with $\boldsymbol{v}_{0}=0$. Those in the non-resonant condition become

$$
\begin{aligned}
v_{x}(t)= & \left(\omega_{B} \omega_{E} / \Omega\right) v_{E} \sin \left(\omega_{E} t^{\prime}+\phi\right) \cos \left[\omega_{B}\left(t-t^{\prime}\right)\right] \\
+ & \left(\omega_{E}^{2} / \Omega\right) v_{E} \cos \left(\omega_{E} t^{\prime}+\phi\right) \sin \left[\omega_{B}\left(t-t^{\prime}\right)\right] \\
& -\left(\omega_{B} \omega_{E} / \Omega\right) v_{E} \sin \left(\omega_{E} t+\phi\right), \\
v_{y}(t)= & \left(\omega_{B} \omega_{E} / \Omega\right) v_{E} \sin \left(\omega_{E} t^{\prime}+\phi\right) \sin \left[\omega_{B}\left(t-t^{\prime}\right)\right] \\
& -\left(\omega_{E}^{2} / \Omega\right) v_{E} \cos \left(\omega_{E} t^{\prime}+\phi\right) \cos \left[\omega_{B}\left(t-t^{\prime}\right)\right]
\end{aligned}
$$


Author Manuscript

$$
+\left(\omega_{E}^{2} / \Omega\right) v_{E} \cos \left(\omega_{E} t+\phi\right) .
$$

Those in the resonant condition are

$$
\begin{aligned}
v_{x}(t)= & {\left[\frac{1}{2} v_{E} \sin \left(\omega t^{\prime}+\phi\right) \cos \left(\omega t^{\prime}+\phi\right)\right.} \\
& \left.+\frac{1}{2} v_{E} \omega\left(t-t^{\prime}\right)\right] \cos (\omega t+\phi) \\
& -\frac{1}{2} v_{E} \cos ^{2}\left(\omega t^{\prime}+\phi\right) \sin (\omega t+\phi), \\
v_{y}(t)= & {\left[\frac{1}{2} v_{E} \sin \left(\omega t^{\prime}+\phi\right) \cos \left(\omega t^{\prime}+\phi\right)\right.} \\
& \left.+\frac{1}{2} v_{E} \omega\left(t-t^{\prime}\right)\right] \sin (\omega t+\phi) \\
& -\frac{1}{2} v_{E} \sin ^{2}\left(\omega t^{\prime}+\phi\right) \cos (\omega t+\phi) .
\end{aligned}
$$

The initial electron population in a squad, $\mathrm{d} n_{\mathrm{sq}}$, is given as $n\left(t^{\prime}\right) \nu d t^{\prime}$ with the total electron population $n\left(t^{\prime}\right)$ at $t^{\prime}$. The decay ratio of the electron population in the squad during $t-t^{\prime}$ is $\exp \left[-\nu\left(t-t^{\prime}\right)\right]$. The integral of $v_{*}(t)$ for all $t^{\prime}$ before the observation time $t$ gives $V_{*}(t)$ ('*' represents $x$ or $y$ ):

$$
V_{*}(t)=\frac{1}{n(t)} \int_{-\infty}^{t} v_{*}(t) \exp \left[-\nu\left(t-t^{\prime}\right)\right] n\left(t^{\prime}\right) \nu d t^{\prime} .
$$

Here, we assume an electron-conservative condition first, and a nonconservative case is explained later. In the electron-conservative condition, $n(t)$ and $n\left(t^{\prime}\right)$ cancel out each other, and the non-resonant solutions of $V_{x}(t)$ and $V_{y}(t)$ are obtained from Eqs. (15), (16), and (19) as

$$
\begin{aligned}
V_{x}(t)= & \frac{2 \nu \omega_{E}^{2} \omega_{B} v_{E} \cos \left(\omega_{E} t+\phi\right)}{\left[\left(\omega_{E}+\omega_{B}\right)^{2}+\nu^{2}\right]\left[\left(\omega_{E}-\omega_{B}\right)^{2}+\nu^{2}\right]} \\
& +\frac{\omega_{E} \omega_{B}\left(\omega_{E}^{2}-\omega_{B}^{2}-\nu^{2}\right) v_{E} \sin \left(\omega_{E} t+\phi\right)}{\left[\left(\omega_{E}+\omega_{B}\right)^{2}+\nu^{2}\right]\left[\left(\omega_{E}-\omega_{B}\right)^{2}+\nu^{2}\right]}, \\
V_{y}(t)= & \frac{\nu \omega_{E}\left(\omega_{E}^{2}+\omega_{B}^{2}+\nu^{2}\right) v_{E} \sin \left(\omega_{E} t+\phi\right)}{\left[\left(\omega_{E}+\omega_{B}\right)^{2}+\nu^{2}\right]\left[\left(\omega_{E}-\omega_{B}\right)^{2}+\nu^{2}\right]} \\
& -\frac{\omega_{E}^{2}\left(\omega_{E}^{2}-\omega_{B}^{2}+\nu^{2}\right) v_{E} \cos \left(\omega_{E} t+\phi\right)}{\left[\left(\omega_{E}+\omega_{B}\right)^{2}+\nu^{2}\right]\left[\left(\omega_{E}-\omega_{B}\right)^{2}+\nu^{2}\right]} .
\end{aligned}
$$

The resonant solutions of $V_{x}(t)$ and $V_{y}(t)$ obtained from Eqs. (17)-(19) agree with the specific forms of Eqs. (20) and (21) at $\omega_{E}=\omega_{B}=\omega$, respectively. Equations (20) and (21) represent both solutions, and $\left(V_{x}(t), V_{y}(t)\right)$ on the $v_{x} v_{y}$-plane draws an ellipse with $t$. This is unchanged even in the case of $\boldsymbol{E} \not \perp \boldsymbol{B}$ and $V_{z} \neq 0$.

Monte Carlo simulations (MCSs) were performed to verify the above derivation, referring to a CCF model gas. ${ }^{35}$ It has a cross section of elastic collision, $q_{\mathrm{el}}$, and those of two kinds of inelastic collisions, $q_{\text {inel, } 1}$ and $q_{\text {inel, } 2}$, defined with electron energy $\varepsilon$ and $\varepsilon_{1 \mathrm{eV}}=1 \mathrm{eV}$ as

$$
\begin{aligned}
& q_{\text {total }}(\varepsilon)=q_{0} / \sqrt{\varepsilon / \varepsilon_{1 \mathrm{eV}}}, \\
& q_{0}=4.0 \times 10^{-19} \mathrm{~m}^{2},
\end{aligned}
$$




$$
\begin{aligned}
& q_{\text {inel }, 1}(\varepsilon)=\left\{\begin{array}{c}
0 \quad \text { for } \varepsilon \leq 10 \mathrm{eV} \\
q_{1}\left[1-\exp \left(-0.25 \times \varepsilon / \varepsilon_{1 \mathrm{eV}}\right)\right]^{10} \\
\times \exp \left[-0.01712 \times\left(\varepsilon / \varepsilon_{1 \mathrm{eV}}-10\right)\right] \\
\text { for } 10 \mathrm{eV}<\varepsilon \leq 300 \mathrm{eV} \\
q_{1} \exp \left[-0.01712 \times\left(\varepsilon / \varepsilon_{1 \mathrm{eV}}-10\right)\right] \\
\text { for } \varepsilon>300 \mathrm{eV}
\end{array}\right. \\
& q_{1}=1.5 \times 10^{-20} \mathrm{~m}^{2}, \\
& q_{\text {inel }, 2}(\varepsilon)=\left\{\begin{array}{c}
0 \quad \text { for } \varepsilon \leq 15 \mathrm{eV} \\
q_{2}\left\{1-\exp \left[-\left(\varepsilon / \varepsilon_{1 \mathrm{eV}}\right)^{0.1}\right]\right\}^{6} \\
\times \exp \left[-0.0015 \times\left(\varepsilon / \varepsilon_{1 \mathrm{eV}}-15\right)\right] \\
\text { for } 15 \mathrm{eV}<\varepsilon \leq 500 \mathrm{eV} \\
q_{2} \exp \left[-0.0015 \times\left(\varepsilon / \varepsilon_{1 \mathrm{eV}}-15\right)\right] \\
\text { for } \varepsilon>500 \mathrm{eV}
\end{array}\right. \\
& q_{2}=2.0 \times 10^{-20} \mathrm{~m}^{2}, \\
& q_{\mathrm{el}}(\varepsilon)=q_{\text {total }}(\varepsilon)-q_{\text {inel }, 1}(\varepsilon)-q_{\text {inel }, 2}(\varepsilon)
\end{aligned}
$$

The collision concerned with $q_{\text {inel,2 }}$ was ionization in Ref. 35 ; however, it was treated as an excitation to test an electron-conservative case at first in the present MCSs. The $\boldsymbol{E} \times \boldsymbol{B}$ fields were set as $E=200 \mathrm{~V} \mathrm{~m}^{-1}, f=13.56 \mathrm{MHz}, \phi=0$, and $B=0.1-1.0 \mathrm{mT}$ with steps of $0.1 \mathrm{mT}$. In addition, $\boldsymbol{V}(t)$ at $B_{\mathrm{ECR}}$ of the rf-resonant value of $B$ was also examined, where $B_{\mathrm{ECR}}=2 \pi f m / e=0.4844 \mathrm{mT}$ at $f=13.56 \mathrm{MHz}$. The $\nu$ was set at $3.82 \times 10^{7} \mathrm{~s}^{-1}$ assuming a gas molecule number density $N=1.61 \times 10^{20} \mathrm{~m}^{-3}$, which corresponds to $0.67 \mathrm{~Pa}$ ( $5 \mathrm{mTorr}$ ) at $300 \mathrm{~K}$, a typical gas pressure in magnetized plasma. ${ }^{27}$ This $\nu$ value is in the range of $f<\nu<\omega_{E}=8.52 \times 10^{7} \mathrm{~s}^{-1}$, and $\omega_{B}$ varies with $B$ from $f<\omega_{B}<\nu$ to $\omega_{E}<\omega_{B}$ through $\omega_{B}=\omega_{E}$. The $\boldsymbol{v}_{0}$ of the initial electrons were chosen at random from a Maxwellian EVDF with $\bar{\varepsilon}=1 \mathrm{eV}$. The time step $\Delta t$ was set at $T / 24000=3.07 \mathrm{ps}$, where $T=1 / f=73.7 \mathrm{~ns}$. The electron flight was calculated using Eq. (3) discretized for every $\Delta t$; Eqs. (7), (8), (11), and (12) were not used in the MCSs so they were independent of the analytical derivation of $\boldsymbol{V}$ as much as possible. Electrons more than $10^{6}$ were tracked for $100 T$, within which $\boldsymbol{V}(t)$ reached its periodic steady state. $\boldsymbol{V}(t)$ values were obtained as the phase-resolved averages in the final $5 T$.

Figure 1 shows the loci of $\boldsymbol{V}(t)$ calculated by Eqs. (20) and (21), and they are verified by the MCS results. The derivation of the analytical solutions is valid. The elliptic locus lies along the $\boldsymbol{E}$ direction under weak $\boldsymbol{B}$ and expands as tilting toward the $\boldsymbol{E} \times \boldsymbol{B}$ direction with increasing $B$. The ellipse becomes the largest around $B=B_{\mathrm{ECR}}$, and shrinks under $B>B_{\mathrm{ECR}}$. This tendency is similar to that in Ar. ${ }^{33}$

The tilt of the major axis of the ellipse relative to the $\boldsymbol{E}$ direction corresponds to 


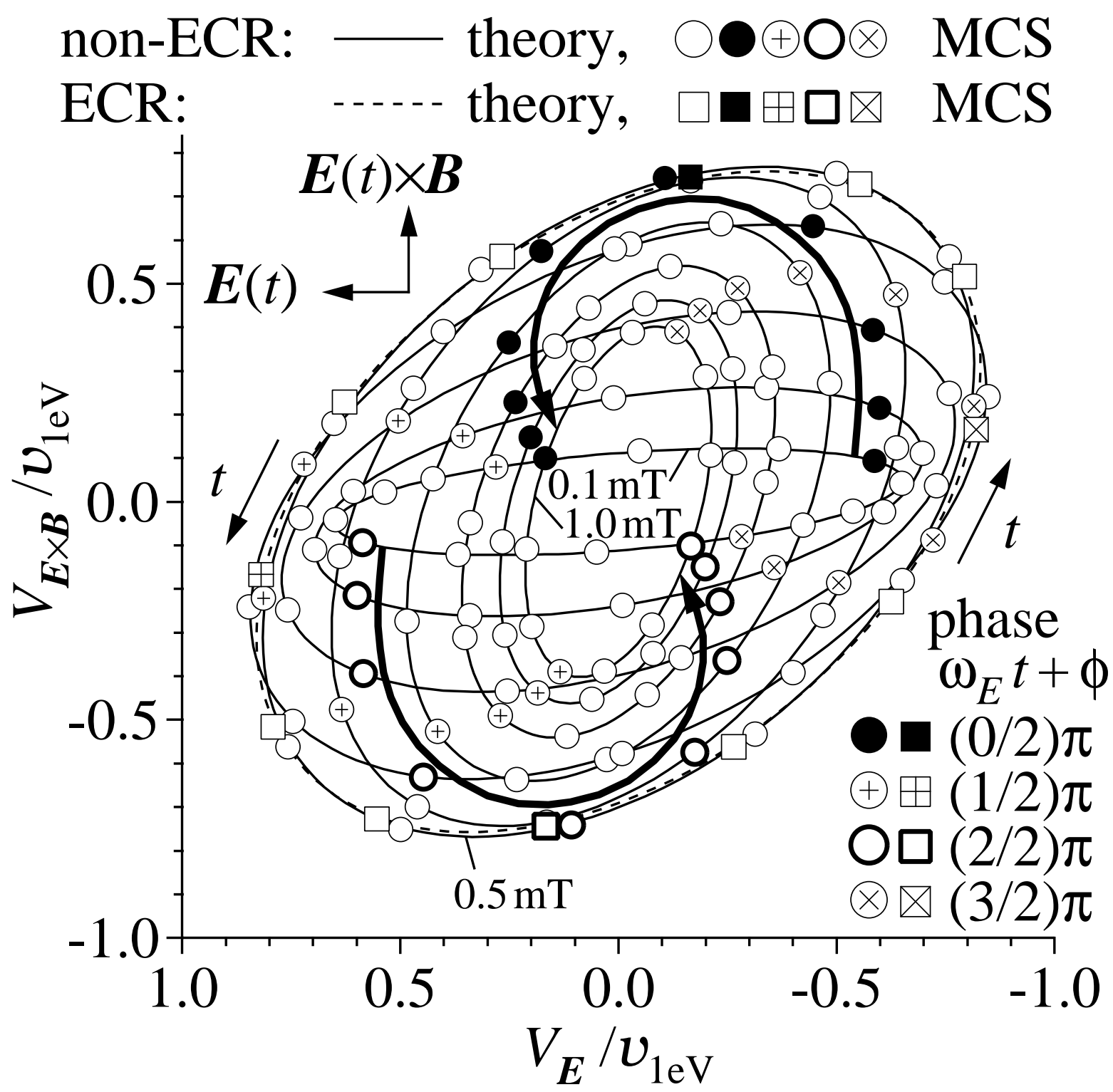

Fig. 1. Vector loci of $\left(V_{\boldsymbol{E}}(t), V_{\boldsymbol{E} \times \boldsymbol{B}}(t)\right)$ for an ac period at $B=0.1-1.0 \mathrm{mT}$ with steps of $0.1 \mathrm{mT}$ and at $B_{\mathrm{ECR}}=0.4844 \mathrm{mT}$. The thick arrows indicate the change of locus with increasing $B$; the locus expands from $0.1 \mathrm{mT}$ to $0.5 \mathrm{mT}$ and shrinks from $0.5 \mathrm{mT}$ to $1.0 \mathrm{mT}$. Curves, theory; and circles and squares, MCS plotted for every $\pi / 6$ of the phase $\omega_{E} t+\phi . V_{\boldsymbol{E}}$ and $V_{\boldsymbol{E} \times \boldsymbol{B}}$ are scaled by the electron speed associated with $1 \mathrm{eV}, v_{1 \mathrm{eV}}$.

the average of the Hall deflection angle $\theta_{\mathrm{H}}=\tan ^{-1}\left(-V_{\boldsymbol{E} \times \boldsymbol{B}} / V_{\boldsymbol{E}}\right),{ }^{33}$ and $V(t)=|\boldsymbol{V}(t)|=$ $\left\{\left[V_{x}(t)\right]^{2}+\left[V_{y}(t)\right]^{2}\right\}^{1 / 2}$ takes its maximum $V_{\max }$ and minimum $V_{\min }$ at the ends of the major and minor axes of the ellipse, respectively. When we rewrite Eqs. (20) and (21) as

$$
\begin{aligned}
& V_{x}(t)=P \cos \left(\omega_{E} t+\phi\right)+Q \sin \left(\omega_{E} t+\phi\right), \\
& V_{y}(t)=R \sin \left(\omega_{E} t+\phi\right)+S \cos \left(\omega_{E} t+\phi\right),
\end{aligned}
$$


$V^{2}$ is represented as

$$
\begin{aligned}
V^{2}= & \frac{1}{2}\left(P^{2}+Q^{2}+R^{2}+S^{2}\right) \\
& +\frac{1}{2}\left(P^{2}-Q^{2}-R^{2}+S^{2}\right) \cos 2\left(\omega_{E} t+\phi\right) \\
& +(P Q+R S) \sin 2\left(\omega_{E} t+\phi\right) .
\end{aligned}
$$

$V_{\max }$ and $V_{\min }$ appear when $d\left(V^{2}\right) / d t=0$, that is,

$$
\begin{aligned}
& \tan 2\left(\omega_{E} t+\phi\right) \\
& \quad=\frac{2(P Q+R S)}{P^{2}-Q^{2}-R^{2}+S^{2}}=\frac{2 \omega_{E} \nu}{\omega_{B}^{2}-\omega_{E}^{2}+\nu^{2}} \equiv K .
\end{aligned}
$$

The $V^{2}$ and $V$ values to give $V_{\max }$ and $V_{\min }$ are

$$
\begin{aligned}
V^{2}= & \frac{1}{2}\left(P^{2}+Q^{2}+R^{2}+S^{2}\right) \\
& \pm \frac{1}{2} \sqrt{\left(P^{2}-Q^{2}-R^{2}+S^{2}\right)^{2}+4(P Q+R S)^{2}} \\
V= & \frac{\frac{1}{2} v_{E} \omega_{E}}{\sqrt{\left(\omega_{E}-\omega_{B}\right)^{2}+\nu^{2}}} \pm \frac{\frac{1}{2} v_{E} \omega_{E}}{\sqrt{\left(\omega_{E}+\omega_{B}\right)^{2}+\nu^{2}}}
\end{aligned}
$$

The positive and negative signs correspond to $V_{\max }$ and $V_{\min }$, respectively. The $V_{\max }$ corresponding to $\bar{\theta}_{\mathrm{H}}$ appears at one of $\omega_{E} t+\phi=\frac{1}{2} \tan ^{-1} K+\frac{1}{2} k \pi(k=0,1,2$, and 3$)$. A case analysis is necessary to specify the quadrant to which the phase $\omega_{E} t+\phi$ corresponding to $\bar{\theta}_{\mathrm{H}}$ belongs.

When $n(t)$ varies by ionization and/or electron attachment (let $\nu_{\text {ion }}$ and $\nu_{\text {att }}$ be their frequencies, respectively) exact derivation of $\boldsymbol{V}(t)$ seems to be difficult because $\varepsilon$ would vary with the ac phase and thus the effective ionization frequency $\nu_{\text {ion }}^{\prime}=\nu_{\text {ion }}-\nu_{\text {att }}$ would not be constant. However, by regarding $\nu_{\text {ion }}^{\prime}$ as a constant, an approximate solution for $\boldsymbol{V}(t)$ is easily obtained. $\mathrm{d} n_{\mathrm{sq}}$ becomes $n\left(t^{\prime}\right)\left(\nu+\nu_{\text {ion }}^{\prime}\right) \mathrm{d} t^{\prime}$ to add the secondary electrons produced by ionization and to subtract the electrons captured by gas molecules. $n(t)$ becomes $n\left(t^{\prime}\right) \exp \left[\nu_{\text {ion }}^{\prime}\left(t-t^{\prime}\right)\right]$ to take its exponential growth into account; thus $n\left(t^{\prime}\right)=n(t) \exp \left[-\nu_{\text {ion }}^{\prime}\left(t-t^{\prime}\right)\right]$. Equation (19) for $V_{*}(t)$ becomes

$$
\begin{aligned}
V_{*}(t) & =\frac{1}{n(t)} \int_{-\infty}^{t} v_{*}(t) \exp \left[-\nu\left(t-t^{\prime}\right)\right] n\left(t^{\prime}\right)\left(\nu+\nu_{\text {ion }}^{\prime}\right) \mathrm{d} t^{\prime} \\
& =\int_{-\infty}^{t} v_{*}(t) \exp \left[-\left(\nu+\nu_{\text {ion }}^{\prime}\right)\left(t-t^{\prime}\right)\right]\left(\nu+\nu_{\text {ion }}^{\prime}\right) \mathrm{d} t^{\prime}
\end{aligned}
$$

This is such a form that $\nu$ in Eq. (19) is replaced with $\nu+\nu_{\text {ion }}^{\prime}$, thus $V_{x}(t)$ and $V_{y}(t)$ in the electron-nonconservative case are obtained by the same replacement for Eqs. (20) and (21), respectively, as well. In additional MCSs in which $q_{\text {inel,2 }}$ was treated as an ionization cross section, $\nu_{\text {ion }}$ reached up to about $10 \%$ of $\nu$ and the vector locus shrank distinguishably from that in the electron-conservative case. This shrinkage was reproduced well by Eqs. (20) and 
(21) modified by the above-mentioned replacement using a time-averaged $\nu_{\text {ion }}$.

The analytical solution of $\boldsymbol{V}(t)$ under ac $\boldsymbol{E}$ and dc $\boldsymbol{B}$ fields in the CCF models has made the basic ac response of the electron drift predictable by clarifying its dependence on $\nu, \omega_{E}$, $E$, and $B$. The vector locus of $\boldsymbol{V}(t)$ visualizes the temporal variations of $\boldsymbol{V}(t)$, and the results of the CCF model will be utilized as a standard to characterize $\boldsymbol{V}(t)$ in real gases.

Acknowledgments This work was supported by KAKENHI Grants JP16K05626 and JP19K03780 from the Japan Society for the Promotion of Science. 


\section{References}

1) T. Uchida, Jpn. J. Appl. Phys. 33, L43 (1994).

2) H. Toyota, K. Takahara, M. Okano, T. Yotsuya, and H. Kikuta, Jpn. J. Appl. Phys. 40, L747 (2001).

3) Y. Morikawa, S. Yasunami, W. Chen, T. Hayashi, and T. Uchida, J. Vac. Sci. Technol. A 19, 1747 (2001).

4) W. Chen, K. Sugita, Y. Morikawa, S. Yasunami, T. Hayashi, and T. Uchida, J. Vac. Sci. Technol. A 19, 2936 (2001).

5) T. Uchida and S. Hamaguchi, J. Phys. D 41, 083001 (2008).

6) T. Tsankov and U. Czarnetzki, IEEE Trans. Plasma Sci. 39, 2538 (2011).

7) T. Tsankov and U. Czarnetzki, AIP Conf. Proc. 1390, 140 (2011).

8) Z. Raspopović, S. Sakadžić, Z. Lj. Petrović, and T. Makabe, J. Phys. D 33, 1298 (2000).

9) Z. M. Raspopović, S. Dujko, T. Makabe, and Z. Lj. Petrović, Plasma Sources Sci. Technol. 14, 293 (2005).

10) S. Dujko, R. D. White, Z. Lj. Petrović, and R. E. Robson, Plasma Sources Sci. Technol. 20, 024013 (2011).

11) S. Dujko, R. D. White, and Z. Lj. Petrović, IEEE Trans. Plasma Sci. 39, 2560 (2011).

12) Y. M. Sung, K. Uchino, K. Muraoka, and T. Sakoda, J. Vac. Sci. Technol. A 18, 2149 (2000).

13) Y. Okraku-Yirenkyi, Y. M. Sung, M. Otsubo, C. Honda, and T. Sakoda, J. Vac. Sci. Technol. A 19, 2590 (2001).

14) T. Sakoda, Y. Okraku-Yirenkyi, Y. M. Sung, M. Otsubo, and C. Honda, Jpn. J. Appl. Phys. 40, 6607 (2001).

15) M. Vural and R. P. Brinkmann, J. Phys. D 40, 510 (2007).

16) H. Sugawara, T. Osaga, H. Tsuboi, K. Kuwahara, and S. Ogata, Jpn. J. Appl. Phys. 49, 086001 (2010).

17) Y. Sakurai, T. Osaga, and H. Sugawara, IEEE Trans. Plasma Sci. 39, 2550 (2011).

18) T. Osaga, H. Sugawara, and Y. Sakurai, Plasma Sources Sci. Technol. 20, 065003 (2011).

19) H. Sugawara, T. Osaga, and H. Yamamoto, Plasma Sources Sci. Technol. 20, 055002 (2011).

20) H. Sugawara, Jpn. J. Appl. Phys. 52, 056101 (2013).

21) Z. Yoshida and T. Uchida, Jpn. J. Appl. Phys. 34, 4213 (1995).

22) H. Asakura, K. Takemura, Z. Yoshida, and T. Uchida, Jpn. J. Appl. Phys. 36, 4493 (1997). 
23) Z. Yoshida, H. Asakura, H. Kakuno, J. Morikawa, K. Takemura, S. Takizawa, and T. Uchida, Phys. Rev. Lett. 81, 2458 (1998).

24) Y. Celik, D. L. Crintea, D. Luggenhölscher, U. Czarnetzki, T. Ishijima, and H. Sugai, Phys. Plasmas 18, 022107 (2011).

25) Y. Minami, Y. Asami, and H. Sugawara, IEEE Trans. Plasma Sci. 42, 2550 (2014).

26) H. Takahashi, K. Nakashima, T. Yamamoto, and H. Sugawara, Jpn. J. Appl. Phys. 57, 126101 (2018).

27) H. Sugawara and S. Ogino, Jpn. J. Appl. Phys. 55, 07LD05 (2016).

28) T. Yamamoto and H. Sugawara, Denki Gakkai Ronbunshi A 137, 363 (2017) [in Japanese].

29) R. Ozawa and H. Sugawara, Denki Gakkai Ronbunshi A 139, 283 (2019) [in Japanese].

30) A. Bogaerts, E. Bultinck, I. Kolev, L. Schwaederlé, K. Van Aeken, G. Buyle, and D. Depla, J. Phys. D 42, 194018 (2009).

31) H. Sugawara, IEEE Trans. Plasma Sci. 47, 1071 (2019).

32) H. Sugawara, Plasma Sci. Technol. 21, 094001 (2019).

33) T. Sato and H. Sugawara, Proc. 34th Int. Conf. Phenomena in Ionized Gases and 10th Int. Conf. Reactive Plasmas, 2019, PO18AM-003.

34) H. Sugawara, T. Yahata, A. Oda, and Y. Sakai, J. Phys. D 33, 1191 (2000).

35) K. Satoh, Y. Ohmori, Y. Sakai, and H. Tagashira, Denki Gakkai Ronbunshi A 111, 198 (1991) [in Japanese]. 